\title{
Dynamic Properties of Angular Vertical Comb- Drive Scanning Micromirrors with Electrothermally Controlled Variable Offset
}

\author{
Ralf Bauer, Li Li, and Deepak Uttamchandani, Senior Member, IEEE
}

\begin{abstract}
This paper presents the investigation of a variable angular vertical comb-drive (AVC) actuated MEMS scanning micromirror, focusing on the influence of the initial comb offset on the dynamic scan characteristics. Continuous control of the fixed comb initial vertical offset is achieved using an electrothermal actuator integrated in the AVC. Electro-thermomechanical simulations and experiments of the AVC structure show good agreement, with the initial vertical offset of the fixed comb reducing theoretically from $10.8 \mu \mathrm{m}(0 \mathrm{~mW}$ to actuator) to $6.0 \mu \mathrm{m}(500 \mathrm{~mW}$ to actuator) and experimentally from $10.4 \mu \mathrm{m}$ (0mW to actuator) to $4.9 \mu \mathrm{m}(510 \mathrm{~mW}$ to actuator). Experimentally, a change of the initial vertical comb-offset from $10.4 \mu \mathrm{m}$ to $4.9 \mu \mathrm{m}$ leads to a reduction of the measured dynamic total optical scan angle (TOSA) from $27^{\circ}$ to $19^{\circ}$ for $100 \mathrm{~V}$ AC actuation. Additionally, a mechanically assisted deflection of the AVC actuators to achieve an almost in-plane comb configuration, further reduces the measured dynamic TOSA to about $3^{\circ}$. The overall device behavior is modeled using a hybrid simulation approach combining FEM analysis of the AVC capacitance with an analytical solution of the motion equation of the scanner. The analysis shows good agreement with the experimentally measured characteristics, overall showing an increased TOSA with higher initial comb offsets.
\end{abstract}

Index Terms-Angular vertical comb-drive (AVC) actuators, scanning micromirrors, electrothermal microactuators, electrostatic force actuators

\section{INTRODUCTION}

$\mathrm{E}$ LECTROSTATIC comb-drive actuated silicon MEMS ${ }_{\text {micromirrors [1] have been the subject of extensive and }}$ detailed research investigations which have led to the application of these devices in optical systems including active corner cube reflectors [2], variable optical attenuators [3, 4], wavelength multiplexers/demultiplexers [5], tunable lasers [6, 7], optical switches [8], as well as micro-projectors and imaging systems [9-13]. An example optical switch system includes an array of $1 \times \mathrm{N}$ comb-drive actuated micromirrors in combination with a grating spectrometer to allow wavelength selective switching [8], while imaging and projection systems

Manuscript received November 5, 2013.

The authors are with the Centre for Microsystems and Photonics, Department of Electronic and Electrical Engineering, University of Strathclyde, Glasgow, G1 1XW, UK (email: ralf.bauer@strath.ac.uk; d.uttamchandani@strath.ac.uk). have been shown, for example, by using in-plane comb-drives creating resonant tilt movements [9] or in-plane rotational movements in combination with a diffraction grating [10]. Imaging and projection systems have also been proposed by using vertical offset comb-drives for actuation of cascaded 1D scanning micromirrors [11] or 2D scanning micromirrors [12, 13]. Asymmetric comb-drive actuators are a subset of general comb-drive actuators. They can generate high torques and are often used for generating stable and wide continuous range of dynamic angular motion, such as required in high scan angle micromirrors. Asymmetric comb-drive actuators are usually classified into two categories, namely staggered vertical combdrives (SVC) and angular vertical comb-drives (AVC). The SVC category actuators have a parallel offset or have a comb finger thickness variation between the fixed and moving combs, while the AVC category actuators have an initial nonzero cross-over angle between the fixed and moving combs.

The fabrication of SVCs in general requires complex multi mask etch steps to create the different comb thickness, either through selective etching $[14,15]$ or by adding isolation layers between two device layers to create the vertical offset between the active comb electrodes [16]. A further fabrication possibility has been demonstrated by using the mechanical displacement of a hinged actuator [13]. For this type of actuator a frequency tuning scheme has also been shown [17] by mechanically increasing the torsional spring stiffness using a chevron actuator. SVC actuators have also been achieved by using bulk micro-machining of a stack of silicon wafers requiring precise alignment between the individual wafers [18].

For AVC actuators various fabrication schemes have been demonstrated including the use of stiction pads [19], the reflow of photoresist [11, 12, 20], plastic deformation [21], mechanical pre-tilt of the torsion beams [22], or the use of non-actuated bimorph layers on the moving [23] or fixed comb structures [24]. In these cases the emphasis has been mainly on investigating the static behavior of the MEMS devices incorporating the AVC actuators and the influence of the comb-drive geometry and initial vertical offsets on the electromechanical characteristics (such as static rotation angles, pullin stabilities or comb-drive capacitances) of the devices when operated at DC voltages. This paper presents an investigation of the dynamic behavior of AVC actuators at different comb 
cross-over angles. Our AVC actuators were realized by exploiting non-uniform stresses, which exist in the silicon device layer of devices fabricated using the SOIMUMPs process [25]. These stresses, combined with thin film stresses arising from the deposition of thin gold layers, generate an outof-plane curvature in cantilever type MEMS structures. This effect has been used advantageously in our work to design AVC actuators with curved stators and rotors, thereby generating a non-zero cross-over angle. Furthermore, we have designed novel AVC actuators whose cross-over angle can be varied by application of an electrical voltage to an electrothermal actuator integrated with the stator of the AVC. We have experimentally and theoretically investigated the dynamic response of such a variable angle AVC by incorporating it in a scanning micromirror, which enables the use of a laser detection system in the experimental characterization. To the best of our knowledge, a detailed investigation of the dynamic response of an AVC actuator for different cross-over angles has not been previously reported.

Theoretical analyses of vertical offset comb-drives have mainly concentrated on the vertical and angular displacements generated when driven by DC voltages. The analytical methods have included electrical field calculations [26] or calculation of the differential capacitance of the comb-drives [23], in both cases ignoring the electrical fringe fields. While for SVC actuators this approach might be valid, the low area overlap of the combs compared to the comb side area of AVCs requires the consideration of fringe fields. This has been achieved in $[11,12,20]$ by use of a hybrid model approach, calculating the differential capacitance through an FEM simulation in combination with an analytical approach to the static torque balance. In their analysis of AVC micromirrors Hong and Syms [2] used a parametric fit of the differential capacitance to match experimentally obtained results without investigating the influence of the initial AVC angle on the dynamic performance. In the work presented here we also use a hybrid approach with a combination of FEM simulation and analytical calculations to obtain the differential capacitance. This has been done for different initial AVC cross-over angles combined with an analytical solution to the dynamic motion equation of the micromirror.

In section II the design and fabrication steps of the AVC scanning micromirror and the novel variable cross-over angle AVC comb actuator are presented. This is followed in section III by the simulation and characterization of the electrothermal actuator used to change the AVC cross-over angle. Section IV presents the experimental characterization of our AVC driven micromirror with varying initial cross-over angle of the stator/rotor combs. The hybrid theoretical model of the scanning micromirror is presented in section $\mathrm{V}$ with a comparison between the experimental and theoretical results in section VI and a concluding discussion in section VII.

\section{DEVICE DESIGN AND FABRICATION}

\section{A. Scanning micromirror design}

The layout of the single crystal silicon (SCS) MEMS scanning micromirror used to investigate the influence of the initial AVC angle on the resonant behavior of the scanner is shown in Fig. 1. The $700 \mu \mathrm{m}$ diameter mirror surface is connected via a $25 \mu \mathrm{m}$ wide circular frame to two rectangular torsion beams, each of length $745 \mu \mathrm{m}$ and width $18 \mu \mathrm{m}$. Similar scanning micromirror designs from our group have shown maximum optical scan angles of around $80^{\circ}$ [7]. The circular frame was chosen to reduce the dynamic mirror distortion and induce a small magnification of the mirror tilt angle in respect to the tilt introduced on the torsion beams [27]. This is achieved by combination with a second set of torsion springs of length $35 \mu \mathrm{m}$ and width $10 \mu \mathrm{m}$ connecting the frame and mirror surface in line with the main torsion springs. The moving combs of the AVC actuator are connected to a single side of one of the torsion beams, while the fixed combs are attached to a comb support beam and anchored through four electrothermal bimorph actuators (see Fig. 1). In total, 13 moving and 14 fixed combs are used to create the comb actuator with each comb finger having a length of $160 \mu \mathrm{m}$ and width of $10 \mu \mathrm{m}$. The lateral gap between the comb electrodes is set to be $6 \mu \mathrm{m}$, which is achieved with high accuracy as both comb electrode structures are defined in the same $10 \mu \mathrm{m}$ thick device layer, as are all other moving structures of the device. Only a single side AVC is used in this device as a further intended use is as an array configuration where generation of a small footprint limits the available space for actuators.

\section{B. Electrothermal variable AVC actuator}

The initial vertical offset of the AVC actuator is defined as the value of $z_{1}$ and $z_{2}$ (see Fig. 4) when the mirror is at its rest position. The offset is generated through stresses in the overall anchor structure of the fixed combs, consisting of the comb support beam, bimorph actuators and anchor pads. A nonuniform stress through the thickness of the silicon device layer creates an out-of-plane curvature of this single side clamped anchor structure. To increase this out-of-plane curvature a

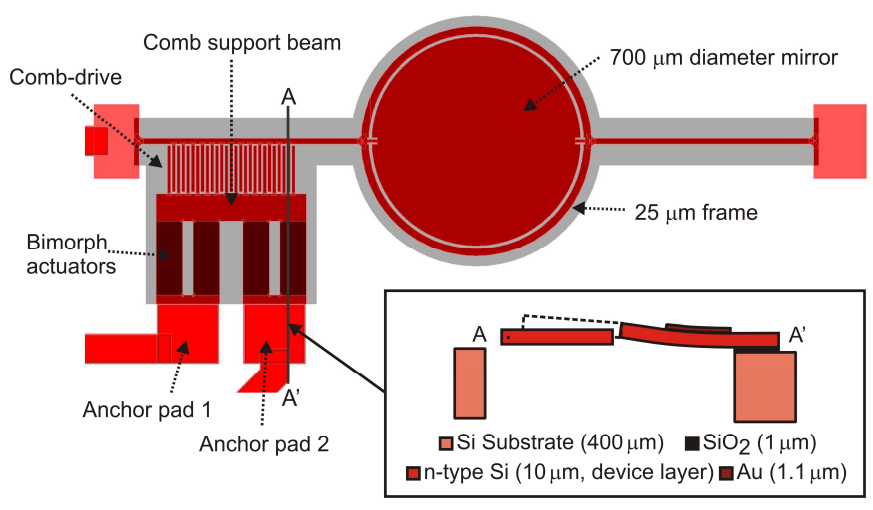

Fig. 1. Schematic layout of AVC scanning mirror including layer structure. 
bimorph layer structure is created on part of the anchor structure by depositing a $1.1 \mu \mathrm{m}$ thick gold coating on its top surface during the microfabrication. These coated bimorph actuators are $250 \mu \mathrm{m}$ long and $88 \mu \mathrm{m}$ wide with two of them connected in parallel to a $90 \mu \mathrm{m}$ wide and $500 \mu \mathrm{m}$ long comb support beam and an anchor pad fixed to the silicon chip substrate (see Figs. 1 and 2).

To enable a continuous variation of the initial vertical offset, an electrothermal actuator is created using the four bimorph beams. With two adjacent bimorph actuators connected in parallel and using a separate electrical connection line, a current path is created through the bimorph anchors and the comb support beam when applying a potential difference between anchor pad 1 and 2 shown in Fig. 1. This current generates Joule heating throughout the current path and the resulting temperature change will change the curvature of the bimorph actuators. Using the material combination of SCS and gold the increase of temperature will lead to a reduction of the curvature and therefore a reduction of the initial vertical offset, $\mathrm{z}_{1}$ and $\mathrm{z}_{2}$. The electrothermal actuator movement range is limited by the maximum temperature in the gold layer to avoid thermal damage, as the analysis of section III will show.

\section{Fabrication process}

The device is fabricated using the commercially available multi-user silicon on insulator process (SOIMUMPs) offered by MEMSCAP Inc [28]. A schematic of the cross-section of the structure is shown in the inset of Fig. 1. All parts of the scanning mirror are defined in the $10 \mu \mathrm{m}$ thick phosphorus doped SCS device layer which is subjected to a single deep reactive ion etch (DRIE) step. The device layer itself is electrically isolated from the $400 \mu \mathrm{m}$ thick SCS substrate by a

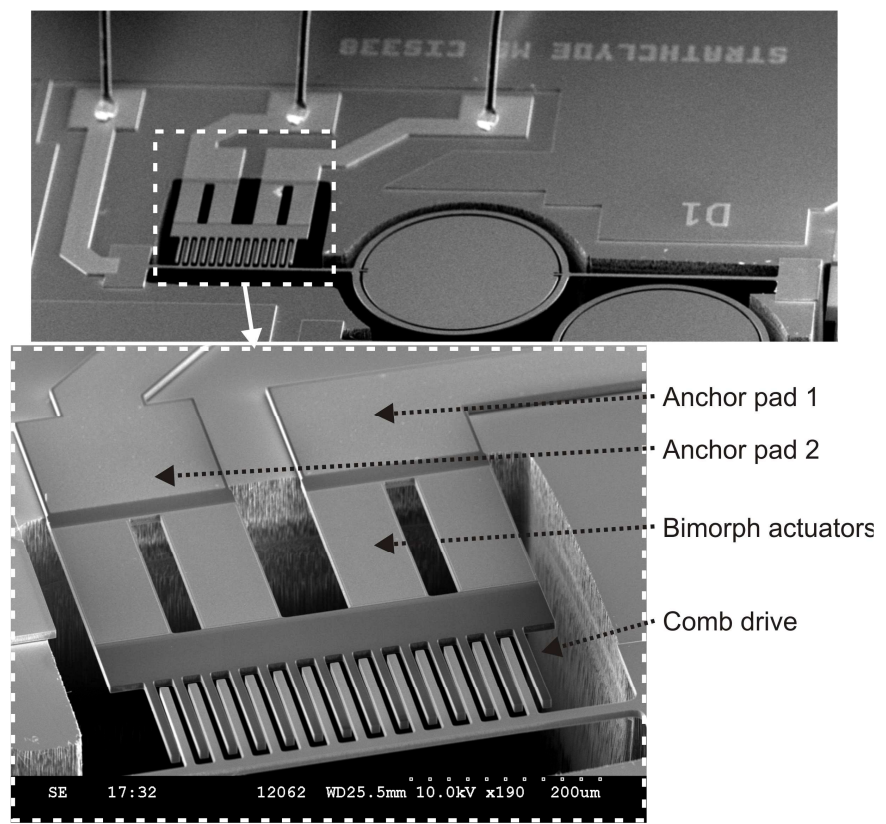

Fig. 2. SEM image of fabricated scanning micromirror incorporating electrothermally controlled variable initial vertical offset AVC actuator.
$1 \mu \mathrm{m}$ thick silicon-dioxide layer. A backside DRIE step is then performed through the thickness of the substrate to release the device. The gold coating layer on the electrothermal actuator beams is produced by a combination of a 500nm thick e-beam evaporated gold layer and a $600 \mathrm{~nm}$ thick shadow mask gold layer, one deposited before and one after the DRIE process steps. A non-uniform stress in the silicon device layer generates an out-of-plane curvature of single side anchored beams, with the free end of a beam being vertically offset above the device layer [25]. This through thickness stressgradient originates from a non-uniform doping profile through the thickness of the silicon device layer. It also leads to an intrinsic curvature of the mirror surface with an experimentally determined radius of curvature of $\sim 0.1 \mathrm{~m}$ [29]. This radius of curvature is outside the usually specified limit of $0.5 \mathrm{~m}$ for mirrors used in imaging or projection systems. However, the desired application of the scanning mirror used in this work is focused on its use as a MEMS based, active intra-cavity device in solid-state laser systems, where optically stable laser cavities can be achieved with the measured mirror surface curvature [7]. An SEM image of the fabricated device is shown in Fig. 2, with a magnified image of the comb-drive in the inset showing the initial vertical offset after fabrication.

\section{ELECTROTHERMAL ACTUATOR SIMULATION AND EXPERIMENTAL CHARACTERIZATION}

\section{A. FEM simulation of the electrothermal actuator}

An electro-thermo-mechanical simulation of the initial vertical offset change of the AVC, achieved through electrothermal actuation of the bimorph actuators of the fixed comb structure, was performed using the FEM software CoventorWare. Using the simulation results, the changing initial vertical offset of the AVC was calculated as the DC voltage applied between the anchor pads was increased. During the simulation, a sweep of varying potential differences

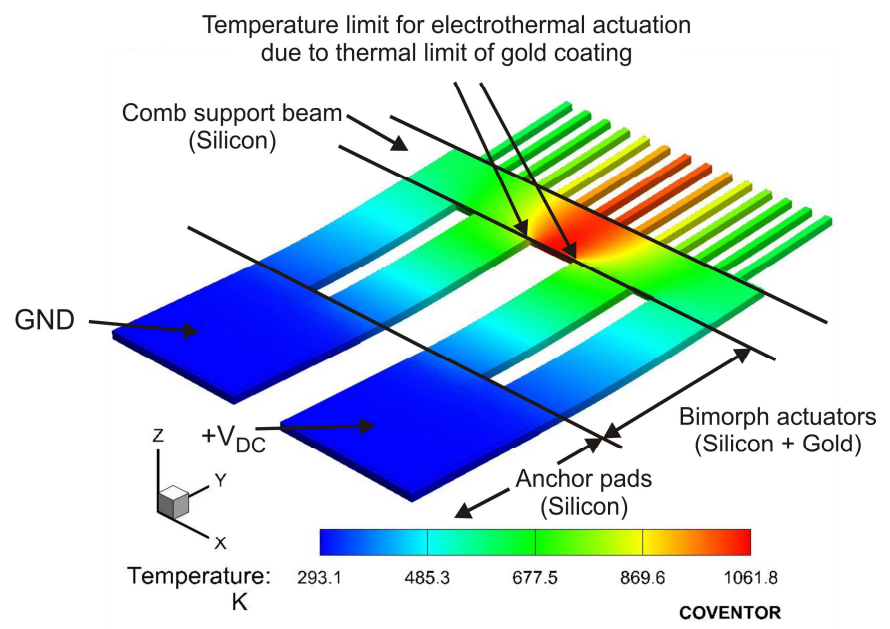

Fig. 3. FEM simulation of electrothermal actuator with a maximum temperature distribution at an applied DC voltage of $3.5 \mathrm{~V}(593 \mathrm{~mW})$ to avoid damage to the gold layers on the anchor beams. 
from $0 \mathrm{~V}$ to $3.5 \mathrm{~V}$ was defined across the faces at the GND and $+\mathrm{V}_{\mathrm{DC}}$ ends of the model (Fig. 3). The simulation software then calculated the temperature profile in the actuator due to Joule heating, heat convection and radiation, and the coupling between thermal and mechanical deformation. The emissivity of the silicon and gold surfaces were taken to be 0.6 and 0.02 respectively. The air convection coefficient for the surfaces of the comb-drives model was taken to be $150 \mathrm{Wm}^{-2} \mathrm{~K}^{-1}$. Other material properties of the silicon and gold layers used for the actuator can be found in previously published work [30]. The intrinsic stress gradient of the silicon layer was $2.4 \mathrm{MPa} / \mu \mathrm{m}$ and the residual stress within the gold layer was taken to be $300 \mathrm{MPa}$. Both effects lead to the out-of-plane curvature of the fixed combs of the comb actuator, while only the intrinsic stress gradient leads to the curvature of the moving combs of the actuator.

The simulated temperature on the surface of the actuator with an applied DC voltage of $3.5 \mathrm{~V}$, leading to an applied DC power of $600 \mathrm{~mW}$, is shown in Fig. 3. The average initial vertical offset $z_{1}$ of the tips of the comb-drives (see inset of Fig. 4) changed from $10.8 \mu \mathrm{m}$ to $5.0 \mu \mathrm{m}$ when the applied power increased from $0 \mathrm{~mW}$ to $600 \mathrm{~mW}$. The simulated change of the initial vertical offsets $z_{1}$ and $z_{2}$ for varying applied DC power is shown in Fig. 4.

\section{B. Experimental characterization of the variable initial vertical offset $A V C$}

To evaluate the performance of the fabricated variable initial vertical offset AVC, the height profile of the structure was measured using a Veeco NT1100 optical white light interferometer profiler. The non-electro-thermally actuated set of combs (i.e. the rotor) of the microscanner were used as the initial height reference, with measurements being taken of the height difference at the end of the fixed combs ( $\mathrm{z}_{1}$, Fig. 4) and the end of the moving combs $\left(\mathrm{z}_{2}\right.$, Fig. 4). The fixed comb support beam (see Fig. 3) showed a maximum height difference of $0.5 \mu \mathrm{m}$ between the middle and the end of the support due to the residual stress in the silicon device layer. A

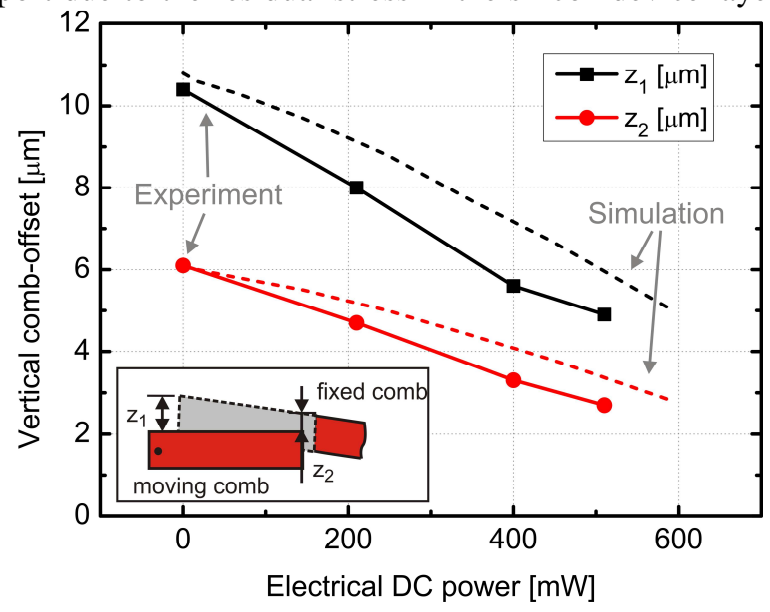

Fig. 4. Average initial vertical offset of comb-drive fingers with electrothermal DC actuation. $z_{1}$ is the offset at the fixed comb end side of the comb-overlap area while $\mathrm{z}_{2}$ is the offset at the moving comb end.
DC voltage was applied between the anchor pads 1 and 2 of the fixed combs, with an electrical power level between $0 \mathrm{~mW}$ and $510 \mathrm{~mW}$ leading to a downward bending of the stator comb tips due to the bimorph effect between the gold and silicon layers of the stator. The fixed comb support beam uniformity changes only minimally during this actuation, showing a maximum height difference change of $0.1 \mu \mathrm{m}$ which did not significantly influence the pre-tilt angle of the AVC.

The measured average initial vertical offsets $z_{1}$ and $z_{2}$ are shown in Fig. 4, with the experimentally obtained trend matching the simulated FEM results. The maximum initial vertical offset $z_{1}$ of $10.4 \mu \mathrm{m}$ is measured without applying an electrical DC power, i.e. this is the value of $z_{1}$ of the device as fabricated. With electrothermal actuation of the bimorph stator, the initial vertical offset reduced to $4.9 \mu \mathrm{m}$ for an applied electrical power of $510 \mathrm{~mW}$. The DC power of the actuator was limited to $510 \mathrm{~mW}$ to avoid thermal damage to the gold coatings of the actuator.

\section{DYNAMIC SCANNER BEHAVIOR WITH VARYING INITIAL VERTICAL OFFSETS OF THE AVC}

The experimental characterization of the influence of the initial vertical offsets $z_{1}$ and $z_{2}$ on the resonant movement behavior of the scanning micromirror was performed using two methods to vary the initial vertical offset, with the combined experimental setup shown in Fig. 5. The first method used a DC voltage control to vary the initial vertical offset as described in Section III. The second method to vary the initial vertical offset was by mechanical displacement of the fixed comb anchor structure using the sharp tip of a needle mounted on a vertical micrometer translation stage. The second displacement method was used to achieve an as close as possible in-plane configuration for the comb-drive and additionally a "negative" initial vertical offset, with the position of the stator combs lying below the plane of the surrounding silicon die, and therefore below the initial position of the rotor combs. A mechanical displacement had to be used for this part as the damage limit of the electrothermal actuator prevented a displacement range leading to an in-plane combdrive configuration. The resulting total optical scan angle (TOSA) for each initial vertical offset of the device was

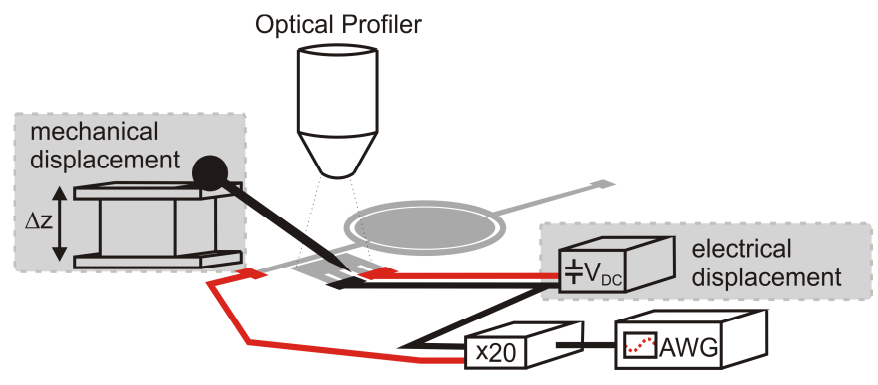

Fig. 5. Experimental setup for mechanical and electrical change of the initial AVC angle with simultaneous evaluation of the initial comb-offset during actuation. Scanner excitation at torsional resonance mode with arbitrary waveform generator (AWG) and high voltage amplifier (x20). 
(a)

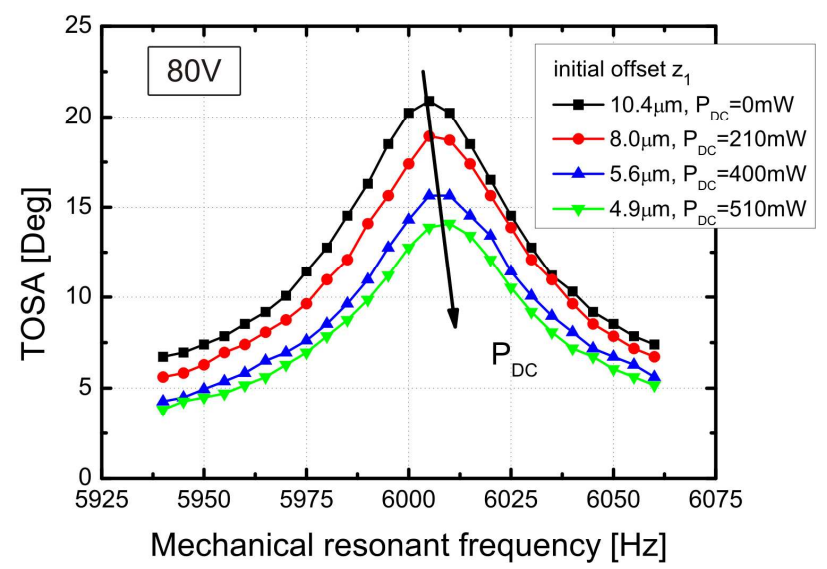

(b)

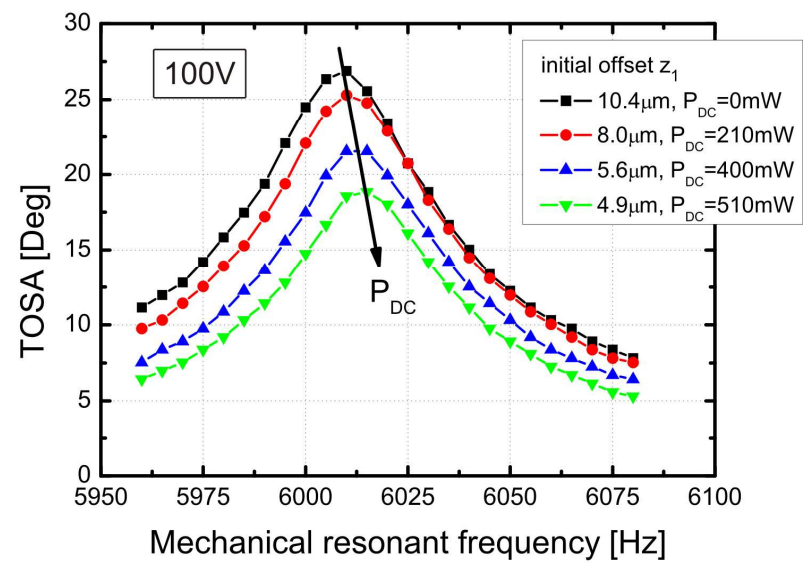

Fig. 6. Frequency response curve of AVC scanning micromirror with electrical variation of the initial comb-offset. (a) Actuation of the scanner with a square-wave voltage signal with $\mathrm{V}=80 \mathrm{~V}$, (b) actuation of the scanner with a square-wave voltage signal with $\mathrm{V}=100 \mathrm{~V}$.

measured using the reflection of a probe HeNe laser beam on the mirror which was projected onto a screen. Simultaneously the initial vertical offsets were measured using a Veeco NT1100 optical white light interferometer profiler.

\section{A. Electrically varied initial vertical offset}

The initial vertical offset was electrically varied while the microscanner was driven at its resonance frequency. A DC power varying from $0 \mathrm{~mW}$ to $510 \mathrm{~mW}$ was applied between the anchor pads 1 and 2 of the stator combs to vary the initial vertical offset as previously explained. The microscanner dynamic displacement was generated using an $\mathrm{AC}$ waveform from a signal generator (Agilent 33250A) which was amplified by a 20x voltage amplifier (FLC A400) before being applied to the scanner. The AC actuation voltages consisted of an offset square-wave waveform with peak-peak value of $80 \mathrm{~V}$ and $100 \mathrm{~V}$ at a frequency of around $6 \mathrm{kHz}$, corresponding to the torsional resonance mode of the scanner. TOSA measurements were taken at four initial vertical offset positions, starting from the maximum initial vertical offset $z_{1}$ of the fixed comb tip of $10.4 \mu \mathrm{m}$ to the lowest possible initial vertical offset of $4.9 \mu \mathrm{m}$ which ensured that the electrothermal actuator was not thermally damaged.

The resulting frequency response curves showing the measured TOSA are presented in Fig. 6. For both values of AC voltages applied to the scanner a reduction of the maximum TOSA is observed for lower values of initial vertical offset. Additionally a resonance frequency increase of $5 \mathrm{~Hz}$ (around $0.08 \%$ ) can be seen as the initial vertical offset is reduced from its maximum value to its minimum value. For $80 \mathrm{~V}$ AC actuation a TOSA of $21^{\circ}$ at $6.005 \mathrm{kHz}$ was measured for the non-actuated initial vertical offset (i.e. without electrothermal actuation) while application of $510 \mathrm{~mW}$ to the fixed comb anchor pads led to a TOSA of $14^{\circ}$ at $6.010 \mathrm{kHz}$. For the $100 \mathrm{~V}$ AC actuation a similar behavior was observed with TOSAs of $27^{\circ}$ at $6.010 \mathrm{kHz}$ and $19^{\circ}$ at $6.015 \mathrm{kHz}$ respectively for the two initial vertical offset values.

\section{B. Mechanically varied initial vertical offset}

The mechanical variation of the initial vertical offset is conducted on the same microscanner as the electrical offset control, creating the vertical displacement of the comb anchors by using a needle tip. The needle is fixed on a vertical micrometer stage and mounted at an angle to allow the simultaneous imaging of the fixed comb displacement using the optical profiler while adjusting the displacement of the combs. The needle tip has a width of around $100 \mu \mathrm{m}$ and is carefully centered between the two sets of bimorph beams to exert a balanced downward pressure leading to a change in initial vertical offset, whilst making great effort to minimize any twisting of the comb structures. Four displacement cases were chosen: (i) initial vertical offset at the fixed comb tip at the rest position of $10.4 \mu \mathrm{m}$ (ii) about half way between the rest position and an in-plane alignment relating to $3.9 \mu \mathrm{m}$ (iii) as close as possible in-plane configuration with an initial vertical offset of $0.5 \mu \mathrm{m}$ and (iv) a negative initial vertical offset of $-1.7 \mu \mathrm{m}$ placing the fixed combs beneath the moving comb rest position. Due to the tip width being smaller than the overall comb-anchor width, the mechanical displacement of the combs leads to a slight non-homogeneous comb tip displacement with higher displacements in the middle compared to the edges of the anchor showing a maximum difference of $1.2 \mu \mathrm{m}$ for the inplane configuration. The quoted offset values are therefore average offsets. 
(a)

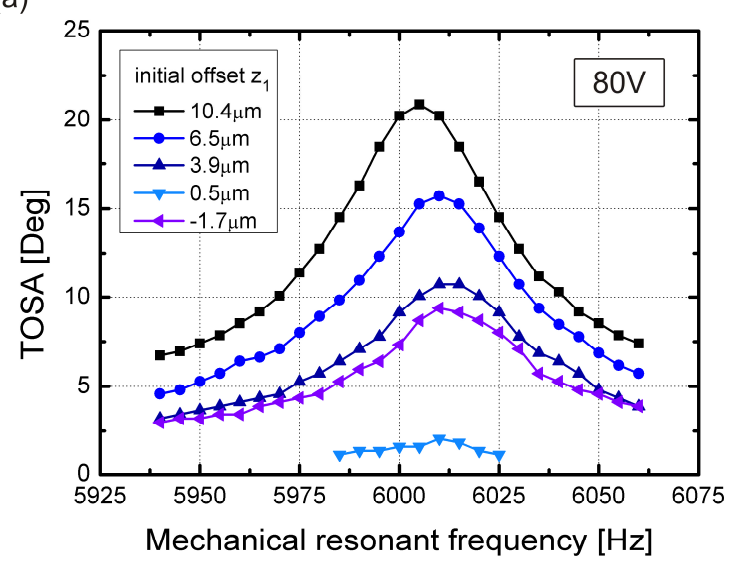

(b)

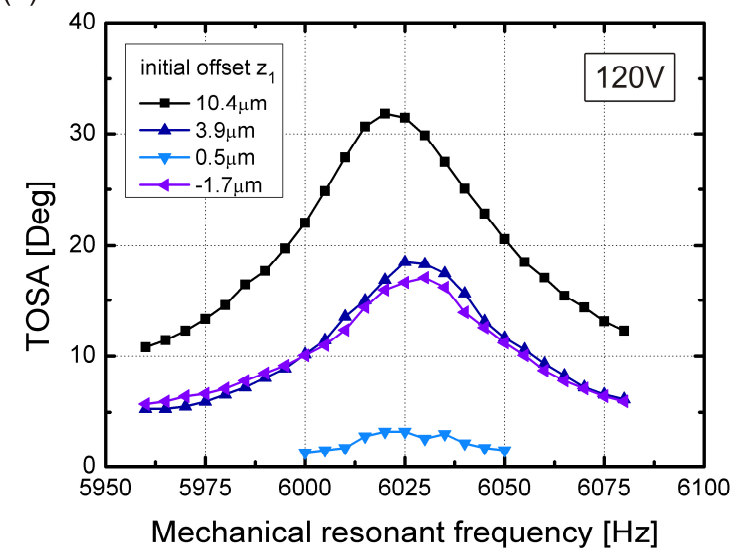

Fig. 7. Frequency response curve of AVC scanning micromirror with mechanical variation of the initial comb-offset using a probing needle. (a) Actuation of the scanner with a square-wave voltage signal with $\mathrm{V}=80 \mathrm{~V}$, (b) actuation of the scanner with a square-wave voltage signal with $\mathrm{V}=120 \mathrm{~V}$.

The frequency response curves for the four cases are shown in Fig. 7 for square-wave $\mathrm{AC}$ actuation voltages of $80 \mathrm{~V}$ and $120 \mathrm{~V}$ peak-peak. Similar to the electrically induced initial vertical offset change, a higher initial vertical offset leads to higher TOSA values at the resonance peak. For $80 \mathrm{~V}$ actuation the TOSA reduces from $21^{\circ}$ at the maximum initial vertical offset to about $2^{\circ}$ for the lowest initial vertical offset difference and rises again to $9^{\circ}$ when reaching the negative initial vertical offset. As already mentioned, no perfect in-plane comb alignment could be reached with the mechanical displacement, leading to an average initial vertical offset of $0.5 \mu \mathrm{m}$ at the tip of the fixed combs $\left(\mathrm{z}_{1}\right)$ and $-0.9 \mu \mathrm{m}$ at the tip of the moving combs $\left(\mathrm{z}_{2}\right)$. This initial vertical offset in both vertical directions plays a part in the low TOSA frequency response for this case. For the $120 \mathrm{~V}$ actuation a similar behavior is observed with the maximum TOSA reducing from $32^{\circ}$ for the initial vertical offset $z_{1}$ to about $3^{\circ}$ for the closest in-plane configuration and rising to $17^{\circ}$ when moving to the negative initial vertical offset. The initial vertical offsets for both actuation voltages are identical since frequency sweeps have been carried out for both without changing the mechanical setup.

Comparing experimental results obtained from both initial vertical offset control techniques shows the same behavior, namely lower absolute values of initial vertical offsets leading to a lower angular response of the microscanner. At the same time a small frequency shift in the region of $5 \mathrm{~Hz}$ towards higher frequencies can be seen at the resonance peaks when moving from the highest initial vertical offsets to the lowest achieved initial vertical offsets.

\section{HYBRID THEORETICAL MODEL FOR AVC SCANNER}

To investigate the expected dynamic behavior of the microscanner for varying initial vertical offsets a theoretical model is used, combining an FEM simulation of the capacitance between the comb fingers of the AVC actuator with the numerical solution of the basic equation of motion of the scanner for torsional displacements. The model for the numerical solution is based on the model used by Hong and Syms [2] for the evaluation of a fixed initial vertical offset AVC. The angular displacement $\theta$ of the scanning mirror around its torsion axis is governed by the equation of motion of a forced oscillator and is written as

$I \ddot{\theta}+2 \xi \omega_{0} I \dot{\theta}+\omega_{0}^{2} I \theta=\frac{1}{2} V^{2}(t) \frac{\partial C(\theta, t)}{\partial \theta}$.

where I is the torsional moment of inertia of the scanner, $\omega_{0}$ is the angular resonant frequency, $\xi$ the damping coefficient, $\mathrm{V}(\mathrm{t})$ the applied voltage driving signal and $\mathrm{C}(\theta, \mathrm{t})$ the angular and time dependent capacitance of the AVC actuator. The damping coefficient is related to the experimentally measured Q-factor of the resonance by

$\xi=\frac{1}{2 Q}=\frac{f_{2}-f_{1}}{2 f_{0}}$,

where $f_{0}$ is the resonance frequency and $f_{2}$ and $f_{1}$ are the frequency values where the angular response has dropped to a value of $1 / \sqrt{2}$ times the maximum angle. The spring related part of the motion equation can be written in form of a torsional spring with spring constant $\mathrm{k}_{0}$ where

$k_{0}=\omega_{0}^{2} I$.

If a nonlinear behavior of the torsional spring is present then this spring constant has to be adapted to include higher order terms, with the major influence being a cubic nonlinearity term for symmetric displacement movements as is present in our microscanner [31]. The modified spring constant then takes the form

$k_{\theta}=k_{0}\left(1+k_{2} \theta^{2}\right)$, 


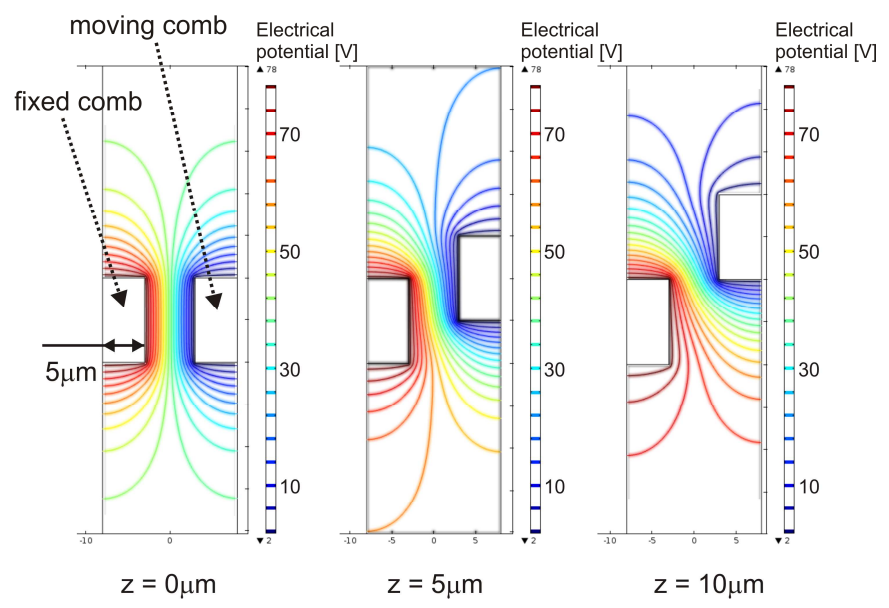

Fig. 8. 2D FEM simulation of electrical equi-potential lines between one pair of half-comb fingers with varying vertical offset for an applied voltage of $\mathrm{V}_{\mathrm{FEM}}=80 \mathrm{~V}$. The electrical energy distribution is used to calculate the capacitance between the comb-fingers including fringe field effects.

where $\mathrm{k}_{\theta}$ is the resulting spring constant and $\mathrm{k}_{2}$ is the cubic nonlinear spring constant. Positive $\mathrm{k}_{2}$ values lead to spring hardening behavior while negative $\mathrm{k}_{2}$ values show a spring softening effect [32]. The right hand side of equation (1) comprises the electrostatic torque applied through the AVC actuator. The electrical actuation voltage can be written as

$$
\begin{aligned}
& V^{2}(t)=\frac{1}{4} V_{0}^{2}[1+\operatorname{sgn}(\sin (2 \pi f t))]^{2}, \\
& \operatorname{sgn}(\sin (2 \pi f t))=\left\{\begin{array}{lll}
+1 & \text { for } & \sin (2 \pi f t)>0 \\
0 & \text { for } & \sin (2 \pi f t)=0 \\
-1 & \text { for } & \sin (2 \pi f t)<0
\end{array}\right.
\end{aligned}
$$

with $\mathrm{V}_{0}$ being the amplitude of the square-wave voltage signal and $f$ the device actuation frequency. To account for the variable capacitance distributions for varying initial vertical offsets and for the fringe fields between the comb electrodes, which are not negligible for AVC actuators [20], a 2D FEM simulation is used combined with an analytical expression for the instantaneous relative comb positions between the rotor and stator.

\section{A. Electrostatic fringe field analysis}

To determine the 2D electrical field distribution for a varying height difference $\mathrm{z}$ of a cross section of the fixed and moving combs in a unit cell of the comb drive a FEM simulation using the software COMSOL Multiphysics is performed. A unit cell consists of the end-on cross section of a half width of a moving and a half width of a fixed comb as shown in Fig. 8. The use of this unit cell reduces the computational requirements, with the use of only a half comb per unit cell to allow the summing of multiple unit cells to calculate the overall comb-drive electrical field distribution [20]. The simulation solves the electrical potential distribution of the unit cell for an applied voltage $\mathrm{V}_{\mathrm{FEM}}$ between both half

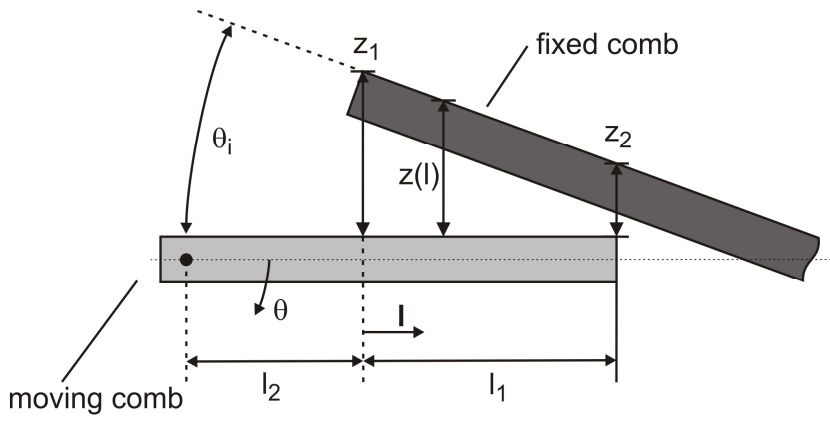

Fig. 9. Geometry definitions for comb-drive overlap calculations of $z(1)$ to calculate the angular dependent differential capacitance.

combs. This is done by defining both half combs as terminals in the FEM software with their respective material properties set to the used SCS. The $6 \mu \mathrm{m}$ gap between the combs is filled with air by using the COMSOL in-built material properties. The electrical energy density $\mathrm{w}_{\mathrm{e}}$ of the unit cell is then calculated using a surface integration of the simulated potential distribution over the 2D area using the FEM software. This process is repeated for varying height steps $\mathrm{z}$ between the two half combs, leading to a distribution of $\mathrm{w}_{\mathrm{e}}$ over varying height differences. The electrical field distribution for three example cross-section offsets of $0 \mu \mathrm{m}, 5 \mu \mathrm{m}$ and $10 \mu \mathrm{m}$ is shown in Fig. 8 (with $\mathrm{V}_{\mathrm{FEM}}=80 \mathrm{~V}$ ), also showing the necessity to include the fringe field in the capacitance determination as major parts of the field distribution are outside the overlap area.

\section{B. Capacitance evaluation over angular movement range}

To determine the capacitance $C(\theta)$ of the actuator for a specific mirror tilt angle $\theta$, with inclusion of the contributions of the fringe fields, the electrical energy density of the $2 \mathrm{D}$ unit cell of section A above needs to be integrated over the length of a comb-finger while taking the instantaneous vertical comb offset $z(1, \theta)$ for each section of the comb fingers into account. The vertical comb offset distribution along the length of the comb-overlap can be formulated using the definitions in Fig. 9. When including the tilt angle $\theta$ of the moving comb it can be written as

$$
z(l, \theta)=l \cdot \sin \left(\theta-\theta_{i}\right)+z_{1}+l_{2} \cdot \sin (\theta) .
$$

1 is the length variable originating from the tip of the fixed comb, $\theta_{\mathrm{i}}$ is the initial angle between the moving and fixed comb, $z_{1}$ is the initial vertical offset at the tip of the fixed comb and $l_{2}$ is the length along the moving comb between the rotation axis and the start of the comb overlap on the fixed comb tip. $\theta_{\mathrm{i}}$ and $\mathrm{z}_{1}$ are inputs which are experimentally obtained from the optical profiler measurements of the fabricated comb-drives. From this expression a calculation of the overall actuator capacitance for a specific tilt angle of the moving combs is achieved using 

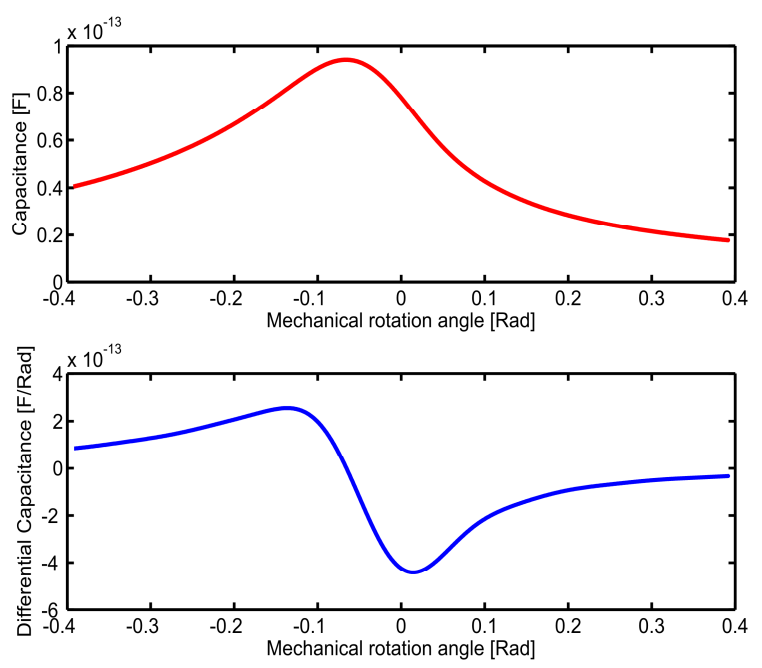

Fig. 10. Calculated capacitance and differential capacitance for an initial AVC offset of $10 \mu \mathrm{m}$ between the fixed and moving combs.

$C(\theta)=N \frac{2}{V_{F E M}^{2}} \int_{0}^{l_{1}} w_{e}(z(l, \theta)) d l$

$\mathrm{N}$ is the number of gaps between the moving and fixed combs and $l_{1}$ the length of the comb overlap, as can be seen in Fig. 9. This integration is undertaken using Matlab with a fit to the electrical energy density distribution and the measured initial comb-offset values as inputs. The differential capacitance $\partial \mathrm{C} / \partial \theta$ is then approximated using

$\frac{\partial C}{\partial \theta} \approx \frac{C(p)-C(p-1)}{\theta(p)-\theta(p-1)}$

with $p$ being the running variable through the calculated set of capacitance values for varying angles $\theta$. An example distribution of the actuator capacitance and differential capacitance for an initial vertical offset $z_{1}$ of $10.4 \mu \mathrm{m}$ is shown in Fig. 10. The considered angular range is in this case an optical scan angle of $\pm 45^{\circ}$.

\section{Analytical motion equation evaluation}

To use the above determined differential capacitance distribution in the torque related term of the motion equation, a $20^{\text {th }}$ order Fourier fit is used on the dataset. A numerical solution of the ordinary differential equation in (1) is constructed using Matlab with its in-built Runge-Kutta solver in the function ODE45. To solve the second order differential equation it needs to be split into two first order equations in the form

$\dot{\theta}_{1}=\theta_{2}$

$\dot{\theta}_{2}=-4 \pi \xi f_{0} \theta_{2}-\frac{k_{\theta}}{I} \theta_{1}+\frac{1}{2 I} V^{2}(t) \frac{\partial C}{\partial \theta_{1}}$

To determine the moment of inertia $\mathrm{I}$ and the spring constant $\mathrm{k}_{0}$ a FEM modal analysis of the scanner layout is used (as seen in Fig. 11), resulting in a value for the moment of inertia of $\mathrm{I}=3.963 \cdot 10^{-16} \mathrm{~kg} \cdot \mathrm{m}^{2}$ and a resonance frequency of $\mathrm{f}_{0}=6255 \mathrm{~Hz}$. This simulated resonance frequency overestimates the experimentally observed frequency of the fabricated device by $250 \mathrm{~Hz}$. Therefore the frequency used as simulation input is based on the experimental results with an initial vertical offset $\mathrm{z}_{1}$ of $10.4 \mu \mathrm{m}$, through which the resonance frequency can be extrapolated as $\mathrm{f}_{0}=5990 \mathrm{~Hz}$, rather than using the FEM value of $6255 \mathrm{~Hz}$. The only other parameter relying on prior measurements is the damping coefficient $\xi$, which is determined through the Q-factor of the frequency response curve when the thermal actuator is not actuated. This experimental estimation is necessary as an accurate analytical calculation of the damping behavior for the micromirror structure is outside the scope of this work. To calculate the frequency response curve, multiple iterations of the ODE45 function are required with varying actuation frequency inputs f. The achievable TOSA for each frequency step is extracted after the simulated oscillation has reached its settling time.

Within the simulation model the influence of the frame and its second pair of torsion beams connecting the mirror surface is neglected and no coupled system of differential equations is constructed. This is justified due to a low amplification factor between the angular displacement of the frame and the mirror which was measured to be below 1.2. The mirror and frame combination can therefore be modeled as a stiff structure in a first order approximation.

\section{VERIFICATION OF SIMULATION RESULTS WITH EXPERIMENTAL DATA}

The simulation results obtained in the previous sections are verified using the experimental data obtained for the $80 \mathrm{~V} \mathrm{AC}$ actuation. Four initial vertical offsets were used, two $z_{1}$ values of $10.4 \mu \mathrm{m}$ and $4.9 \mu \mathrm{m}$ for comparison with the electrothermally varied initial vertical offsets, and two $\mathrm{z}_{1}$ values of $0.5 \mu \mathrm{m}$ and $-1.7 \mu \mathrm{m}$ for comparison with the mechanically obtained initial vertical offsets. A summary of

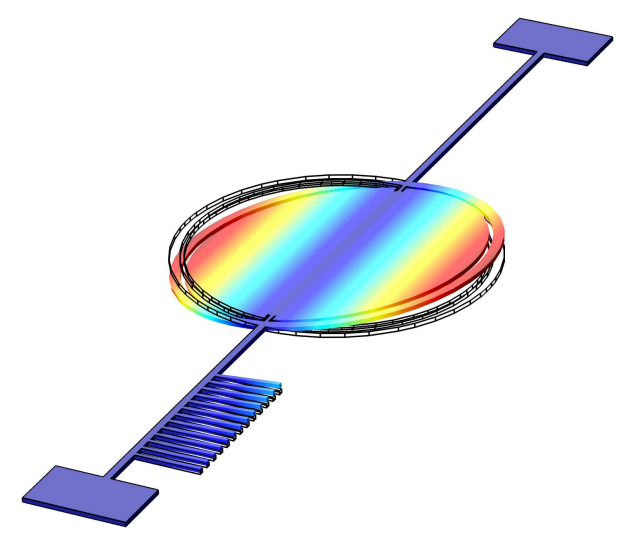

Fig. 11. FEM simulation of torsion resonance mode shape. 
the input parameters for the simulation is presented in Table 1, with the spring constant $\mathrm{k}_{0}$ being calculated through the modal FEM results for $I$ and $f_{0}$. The two initial vertical offset parameters $z_{1}$ and $z_{2}$ for each offset case are the average values taken from the optical profiler measurements and are used for determining the differential capacitance distribution. The damping coefficient is taken from the $10.4 \mu \mathrm{m}$ initial vertical offset frequency response curve of the electrical displacement case in Fig. 6(a) with a Q-factor of 166. The actuation voltage $\mathrm{V}_{0}$ used in the simulation model is $56 \mathrm{~V}$. A cubic nonlinear spring constant of $\mathrm{k}_{2}=0.33$ was included in the modeling as the experimental measurements of the TOSAs showed no nonlinear characteristics (e.g. bending of the frequency response curve) while an initial simulation showed a nonlinear response at movement angles above $\operatorname{TOSA}=15^{\circ}$. This resulted from electrostatic field induced nonlinearities in the simulation, which were counteracted by modifying the simulation to include the nonlinear behavior of the torsional spring.

The resulting simulated frequency response curves for the four actuation cases are shown as lines in Fig. 12, with the open points depicting the experimentally obtained values from section IV. The simulation results show a good agreement with the experimental values with the trend of lower TOSA for lower initial vertical offsets being also present in the simulation results. The experimentally observed frequency shift for lower initial vertical offsets is also shown by the simulation results, with the simulation having a shift of $20 \mathrm{~Hz}$ between the initial vertical offset of $10.4 \mu \mathrm{m}$ and the closest inplane configuration with $0.5 \mu \mathrm{m}$ and therefore introducing an even higher shift than experimentally observed.

\section{CONCLUSION}

Both the experimental and simulation results have shown higher dynamic scan angles of the AVC actuated scanning

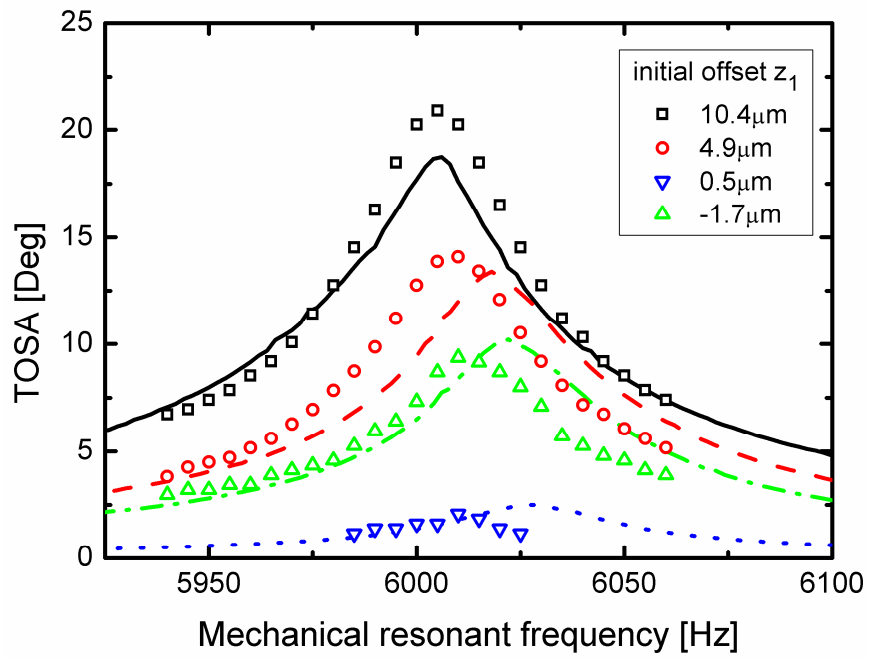

Fig. 12. Comparison of the simulated resonance curves and the measurement results for $80 \mathrm{~V}$ actuation. $10 \mu \mathrm{m}$ and $5 \mu \mathrm{m}$ offset measurements are from the electrical offset actuation and $0 \mu \mathrm{m}$ and $-2 \mu \mathrm{m}$ offset measurements are from the mechanical offset actuation.
TABLE I

PARAMETER OF MOTION EQUATION SIMULATION

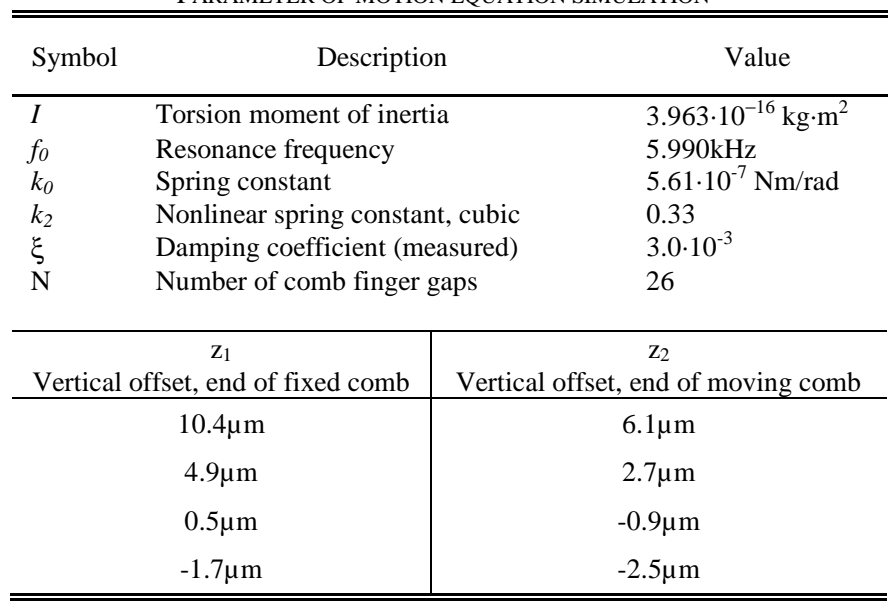

micromirrors for higher initial vertical offsets. The AVC scanning micromirrors are themselves novel in design and operation, since the relative position of the stator and rotor combs can be changed by electrical control. Therefore, the comb-offset for the AVC can be adjusted precisely. No perfect in-plane configuration can be achieved between the stator and rotor combs; however a low initial fixed comb angle of $0.5^{\circ}$ is the closest experimental realization of an in-plane configuration. The full set of possible initial vertical offsets for our AVC microscanners were investigated, with the maximum possible initial vertical offset limited in order to prevent thermal damage of the gold layer coating the electrothermal actuator. Alternative designs for the fixed comb anchor actuator may increase the possible initial vertical offset variation range and could lead to the determination of a maximum initial vertical offset range after which the dynamic scanner behavior will not have a further increase in the angular frequency response. The hybrid simulation model presented shows a good agreement with the experimental data with an already mentioned overestimation of the frequency shift for changing maximum TOSAs. Only a cubic nonlinearity is used for modeling the spring constant with a possible higher agreement between the experimental and simulated data when considering further orders of nonlinearity. These would require an optimization for a fit of the simulations to the experimental data, which was not conducted in the model developed as apart from the damping coefficient, all other model parameters were based on theoretical values.

Overall, an investigation of the dynamic behavior of AVC actuated microscanners has been presented, with the focus on studying the impact of the initial vertical offset of the actuators on the dynamic scan angles generated. Similar to previous investigations of the static scan angles, a higher initial vertical offset was shown to give a higher angular dynamic response at resonance. Experimental results matched the theoretical findings of a hybrid simulation model.

\section{ACKNOWLEDGMENT}

The authors would like to thank Mr. James Gordon Brown 
for fruitful discussions; R.B. would also like to acknowledge funding from the Scottish Founding Council.

\section{REFERENCES}

[1] R. Bauer, G. Brown, L.Li, and D. Uttamchandani, "A novel continuously variable angular vertical comb-drive with application in scanning micromirror," in IEEE 26th International Conference on Micro Electro Mechanical Systems, pp. 528-531, January 2013.

[2] Y. K. Hong and R. R. A. Syms, "Dynamic response modeling of MEMS micromirror corner cube reflectors with angular vertical combdrives," Journal of Lightwave Technology, vol. 25, no. 2, pp. 472-480, Feb. 2007.

[3] T.-S. Lim, C.-H. Ji, C.-H. Oh, H. Kwon, Y. Yee, and J. Bu, "Electrostatic MEMS variable optical attenuator with rotating folded micromirror," IEEE Journal of Selected Topics in Quantum Electronics, vol. 10, no. 3, pp. 558-562, 2004.

[4] L. Li and D. Uttamchandani, "Twin-bladed microelectro mechanical systems variable optical attenuator," Optical Review, vol. 13, no. 2, pp. 93-100, 2006.

[5] Y. Liu, J. Xu, S. Zhong, and Y. Wu, "Large size MEMS scanning mirror with vertical comb drive for tunable optical filter", Optics and Lasers in Engineering, vol. 51, no. 1, pp. 54-60, 2013.

[6] X. M. Zhang, A. Q. Liu, D. Y. Tang, and C. Lu, "Discrete wavelength tunable laser using microelectromechanical systems technology," Applied Physics Letters, vol. 84, no. 3, pp. 329-331, 2004.

[7] R. Bauer, W. Lubeigt, and D. Uttamchandani, "Dual Q-switched laser outputs from a single lasing medium using an intracavity MEMS micromirror array," Optics Letters, vol. 37, no. 17, pp. 3567-3569, Aug. 2012.

[8] D. Hah, S. T.-Y. Huang, J.-C. Tsai, H. Toshiyoshi, and M. C. Wu, "Low-voltage, large-scan angle MEMS analog micromirror arrays with hidden vertical comb-drive actuators," Journal of Microelectromechanical Systems, vol. 13, no. 2, pp. 279-289, Apr. 2004.

[9] H. Schenk, T. Sandner, C. Drabe, T. Klose, and H. Conrad, "Single crystal silicon micro mirrors," Physica Status Solidi C, vol. 6, no. 3, pp. 728- 735, 2009.

[10] Y. Du, G. Zhou, K. L. Cheo, Q. Zhang, H. Feng, and F. S. Chau, "Double-layered vibratory grating scanners for high-speed highresolution laser scanning," Journal of Microelectromechanical Systems, vol. 19, no. 5, pp. 1186-1196, Oct. 2010.

[11] M. Fujino, P. R. Patterson, H. Nguyen, W. Piyawattanametha, and M. C. Wu, "Monolithically cascaded micromirror pair driven by angular vertical combs for two-axis scanning," IEEE Journal of Selected Topics in Quantum Electronics, vol. 10, no. 3, pp. 492-497, May 2004.

[12] W. Piyawattanametha, P. R. Patterson, D. Hah, H. Toshiyoshi, and M. C. Wu, "Surface- and bulk- micromachined two-dimensional scanner driven by angular vertical comb actuators," Journal of Microelectromechanical Systems, vol. 14, no. 6, pp. 1329-1338, Dec. 2005.

[13] T. Sandner, D. Jung, D. Kallweit, T. Grasshoff, and H. Schenk, "Microscanner with vertical out of plane combdrive," in 16th International Conference on Optical MEMS and Nanophotonics, pp. 33-34, 2011.

[14] D. Lee, U. Krishnamoorthy, K. Yu, and O. Solgaard, "Single-crystalline silicon micromirrors actuated by self-aligned vertical electrostatic combdrives with piston-motion and rotation capability," Sensors and Actuators A: Physical, vol. 114, no. 2-3, pp. 423-428, Sep. 2004.

[15] V. Milanovic, S. Kwon, and L. P. Lee, "High aspect ratio micromirrors with large static rotation and piston actuation," IEEE Photonics Technology Letters, vol. 16, no. 8, pp. 1891-1893, Aug. 2004.

[16] E. T. Carlen, S. Bakshi, A. Pareek, and C. H. Mastrangelo, "High-aspect ratio vertical comb-drive actuator with small self-aligned finger gaps," Journal of Microelectromechanical Systems, vol. 14, no. 5, pp. 11441155, Oct. 2005.

[17] J.-I. Lee, S. Park, Y. Eun, B. Jeong, and J. Kim, "Resonant frequency tuning of torsional microscanner by mechanical restriction using MEMS actuator," in IEEE 22nd International Conference on Micro Electro Mechanical Systems, pp. 164-167, 2009.

[18] Y. Li, D. Psychogiou, S. Kuhne, J. Hesselbarth, C. Hafner, and C. Hierold, "Large stroke staggered vertical comb-drive actuator for the application of a millimeter-wave tunable phase shifter," Journal of Microelectromechanical Systems, vol. 22, no. 4, pp. 962-975, Aug. 2013.

[19] M. Yoda, K. Isamoto, C. Chong, H. Ito, A. Murata, S. Kamisuki, M. Atobe, and H. Toshiyoshi, "A MEMS 1-D optical scanner for laser projection display using self-assembled vertical combs and scan-angle magnifying mechanism," in 13th International Conference on SolidState Sensors, Actuators and Microsystems, pp. 968-971, 2005.

[20] D. Hah, P. R. Patterson, H. D. Nguyen, H. Toshiyoshi, and M. C. Wu, "Theory and experiments of angular vertical comb-drive actuators for scanning micromirrors," IEEE Journal of Selected Topics in Quantum Electronics, vol. 10, no. 3, pp. 505-513, May 2004.

[21] J. Kim, H. Choo, L. Lin, and R. S. Muller, "Microfabricated torsional actuators using self-aligned plastic deformation of silicon," Journal of Microelectromechanical Systems, vol. 15, no. 3, pp. 553-562, Jun. 2006.

[22] Y. Eun, H. Na, J. Choi, J. Lee, and J. Kim, "Angular vertical comb actuators assembled on-chip using in-plane electrothermal actuators and latching mechanisms," Sensors and Actuators A: Physical, vol. 165, no. 1, pp. 94-100, Jan. 2011.

[23] H. Xie, Y. Pan, and G. K. Fedder, "A CMOS-MEMS mirror with curled-hinge comb drives," Journal of Microelectromechanical Systems, vol. 12, no. 4, pp. 450-457, Aug. 2003.

[24] P. Qu and H. Qu, "A novel CMOS-MEMS scanning micro-mirror using vertical comb drives," in 17th International Conference on Optical MEMS and Nanophotonics, pp. 63-64, 2012.

[25] D. Miller, B. Boyce, M. Dugger, T. Buchheit, and K. Gall, "Characteristics of a commercially available silicon-on-insulator MEMS material," Sensors and Actuators A, vol. 138, no.1, pp. 130-144, 2007.

[26] J.-L. A. Yeh, C.-Y. Hui, and N. C. Tien, "Electrostatic model for an asymmetric combdrive," Journal of Microelectromechanical Systems, vol. 9, no. 1, pp. 126-135, Mar. 2000.

[27] A. Arslan, D. Brown, W. O. Davis, S. Holmström, S. K. Gokce, and H. Urey, "Comb-actuated resonant torsional microscanner with mechanical amplification," Journal of Microelectromechanical Systems, vol. 19, no. 4, pp. 936-943, 2010

[28] Memscap Inc., 12 Alexander Drive, Building 100, Research Triangle Park, NC 27709, USA, www.memscapinc.com

[29] R. Bauer, G. Brown, and D. Uttamchandani, "Comparison of the curvature homogeneity and dynamic behaviour of framed and frameless electrostatic X/Y scanning micromirrors," Micro \& Nano Letters, vol. 6, no. 6, pp. 425-428, 2011.

[30] L. Li, R. Li, W. Lubeigt, and D. Uttamchandani, "Design, simulation, and characterization of a bimorph varifocal micromirror and its application in an optical imaging system," Journal of Microelectromechanical Systems, vol. 22, no. 2, pp. 285-294, 2012.

[31] M. Palaniapan and L. Khine, "Nonlinear behavior of SOI free-free micromechanical beam resonator," Sensors and Actuators A: Physical, vol. 142, no. 1, pp. 203-210, Mar. 2008.

[32] A. H. Nayfeh and D. T. Mook, Nonlinear Oscillations, New York: Wiley, 1979, pp. 161-257.

Ralf Bauer received the Dipl.-Ing. degree in Mechatronics from the University of Erlangen-Nuernberg, Germany in 2010, and the Ph.D. degree from the University of Strathclyde, Glasgow, U.K., for work on MEMS micromirrors as active intra-cavity devices in solid-state laser systems, in 2013.

$\mathrm{He}$ is currently a Post-doctoral Research Associate in the Centre for Microsystems and Photonics, University of Strathclyde, working on integration of MEMS devices in miniature photoacoustic spectroscopy gas sensors and optical sensors for trace gas detection. His research interests include the development and integration of MEMS in optical systems and laser systems.

Dr. Bauer is a member of the Optical Society and former vice-president and current committee member of the University of Strathclyde student chapter of the OSA, IOP and EPS. 
$\mathbf{L i} \mathbf{L i}$ received a joint B.Eng. degree in electronic and electrical engineering from both North China Electric Power University, Beijing, China, and the University of Strathclyde, Glasgow, U.K., in 2008, and then a M.Sc. degree in control, communication, and digital signal processing from the University of Strathclyde, in 2009. In 2013, she obtained a PhD degree for her research in the design, characterization, and FEM simulation of MEMS devices in the Centre for Microsystems and Photonics in University of Strathclyde.

She is now working for the CNOOC research institute as a research and design enigineer in Beijing, China.

Deepak Uttamchandani (SM'05) received the Ph.D. degree from University College London, London, U.K., in the area of optical fiber sensors, in 1985.

$\mathrm{He}$ is currently the Director of the Centre for Microsystems and Photonics, University of Strathclyde, Glasgow, U.K. His early research in MEMS concentrated on optothermal microresonator sensors and in investigating techniques for general MEMS material characterization using MEMS micromechanical resonators. His recent research has concentrated on developing system applications of optical MEMS such as intracavity MEMSbased laser systems, MEMS-based photoacoustic spectroscopy for gas sensing, and MEMS-based single-pixel imaging systems. He has also published in the field of subwavelength tip-based Raman spectroscopy, which has contributed to the development of tip-enhanced Raman spectroscopy and in the area of in situ intraocular drug detection systems via optical spectroscopy in the living eye. 